\title{
Interactive comment on "Photochemical
} degradation of iron(III)-citrate/citric acid aerosol quantified with the combination of three complementary experimental techniques and a kinetic process model” by Jing Dou et al.

Jing Dou et al.

jing.dou@env.ethz.ch

Received and published: 3 November 2020

We reply to the comments of reviewers 2, 3 and 4 in the attached document. On behalf of all authors, Ulrich Krieger

Please also note the supplement to this comment: 
Interactive comment 